\title{
Advances in basic nuclear science associated with nuclear energy data
}

\author{
H. Flocard \\ CSNSM, IN2P3, CNRS, Université Paris Sud, Bât. 108, 91405 Orsay Campus, France
}

\begin{abstract}
Using few examples, this document attempts to illustrate the connexions between the physics, the methods both theoretical and experimental used in the collection of data relevant for Nuclear Energy and those of basic Nuclear Physics. The specialisation on themes and subjects imposed by an applicative science certainly generates overall shifts in focus as well as originality in techniques in order to meet the specific needs of the engineer. On the other hand, the contact always remains close with the most fundamental nuclear science which both nourishes, and is nourished by the Nuclear Energy Data enterprise.
\end{abstract}

\section{Introduction}

An accurate determination of the cross sections of the set of reactions induced by neutrons on the elements contained in the fuel of present and future reactors is the basis of a true understanding of the workings of these machines. Moreover, it is crucial to extend the associated studies to neighbour nuclei generated by the transmutations through successive captures followed by diverse types of decay ( $\alpha, \beta, \gamma$, fission). The need for accuracy is determined by the principle and by the operating mode of the reactor and thus by the nature and the quantities of products which appear during the operation. However, the ever growing importance of numerical simulation gives an important weight to evaluated data bases such as JEFF, JNDL or ENDF. Since the quality of such bases depends in great part on their internal coherence over a large network of nuclei and reactions around the major isotopes of the nuclear cycles, it is necessary to rely on a set of data which extends beyond that which at first glance would seem necessary. One may say that it is only the theory-experiment coherence in the data bases which sets the precision requirements for any reaction. In this field, quality must be understood global. Only this will allows one to overcoming the effect of uncertainties in measurements as well as the uneven compatibility of distinct experimental results. In particular, it is crucial that experimental uncertainties not exceed those associated with the theories which incorporate the data into the evaluation process.

In a region of the nuclear chart where the number of isotopes whose half life is long compared with the time intervals set by present experimental constraints, can be counted on the fingers of both hands, a direct determination of cross sections at the desired level of accuracy may turn out to be an impossible task. In this text, we shall first consider neutron induced reactions. There, difficulties come up everywhere. Production of a neutron beam of controlled quality is only realized in a limited number of facilities (not more than two to three on each continent). This seriously limits experimental opportunities. Production methods are often plagued by parasite phenomena which one has to protect the experiments from, generally at the cost of a significant reduction of fluxes. Making the required targets often is a major problem. Nowadays, most of them cannot be manufactured either because the material is not available in required form or because fabrication would either lead to violations of safety requirements process or unacceptable costs. In a world which, for very good reasons, is ever more concerned by the risk to personal, safety also restricts installation of such targets at most facilities. Finally, even detection can face major problems when the background generated either by the scattered neutrons, the parasite phenomena or the radioactive decays from the target overlaps with the signal in the detectors.

\section{Transfer reactions}

Confronted with such a situation, experimentalists generally turn to indirect methods whenever a characteristic of the physical process allows one to do it. For low energy neutron induced reactions, the Compound Nucleus $(\mathrm{CN})$ stage offers such an opportunity. In its most elementary version advanced by Bohr just before the second world war, the absorption of the neutron, is followed by a "digestion" phase leading to a new excited nuclear system with a mass increased by one unit. Then, this CN system decays using all the channels (capture, fission, particle emission) which are open at that energy.

This existence of such a two-step process is the main justification advanced for the "surrogate" method. It assumes that the theoretical understanding of the $\mathrm{CN}$ formation cross section is under control and it focuses the experimental task on the determination of the various decay processes (fission, capture...). On the other hand, to generate the $(\mathrm{A}+1) \mathrm{CN}$, the method relies on a combination of beams and targets which is easier to handle. For instance to study fission following the reaction $\left({ }^{233} \mathrm{Am}+\mathrm{n} \rightarrow{ }^{234} \mathrm{Am} *\right)$ which plays an important role in the Thorium cycle, one will instead consider the reaction $\left({ }^{232} \mathrm{Th}+{ }^{3} \mathrm{He} \rightarrow \mathrm{p}+{ }^{234} \mathrm{Am} *\right)$. The advantages of this method are obvious. Target and beams are easier to manufacture and manipulate. Moreover the detection of a charged particle such as a proton and the measure of its energy are comparatively easier. In a single experiment, the surrogate method yields information on a range of excitation energies of the $\mathrm{CN}$. Detection is also facilitated because kinematical conditions 


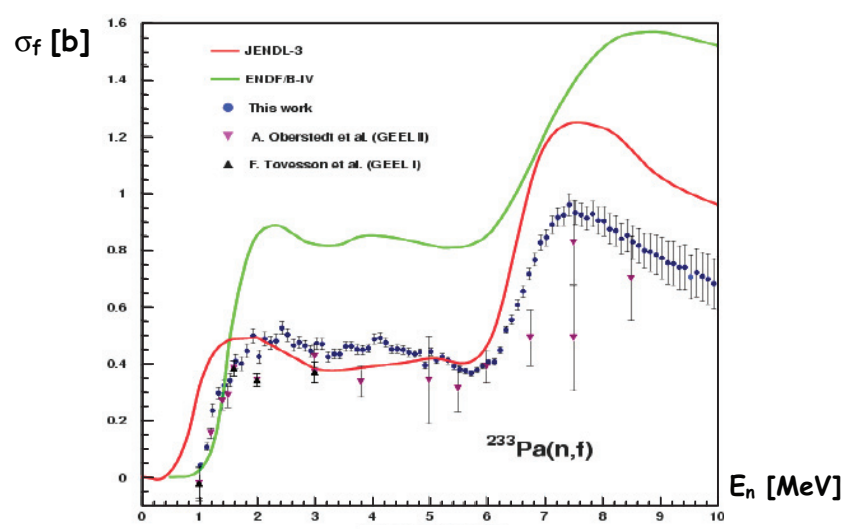

Fig. 1. Fission cross section (in barn) following a n-capture by ${ }^{233} \mathrm{Am}$ as a function of neutron energy over the range [0-10 MeV]. Surrogate method results are shown in blue [1] while red points are those of a direct measurement $[7,8]$. Solid lines correspond to evaluated data bases values $(\mathrm{red}=\mathrm{JENDL}$, green $=\mathrm{ENDF})$.

can be monitored while background noise is reduced. The main difficulty (not specific to this method) lies in the detector equipment efficiency calibration. With the same experimental conditions, using an alternative detection system opens the possibility to study another decay channel of the $\mathrm{CN}$. For instance, using the reaction mentioned above it was possible to determine both the fission and the capture-plus- $\gamma$ de-excitation cross-sections following the capture of a neutron by ${ }^{233} \mathrm{Am} \mathrm{[1,2].} \mathrm{Figure} 1$ shows the results for the fission channel.

Another interest of the surrogate method is the possibility to explore several reactions with the same set-up. Indeed, according to the particle which is detected ( $\mathrm{p}, \mathrm{d}, \mathrm{t}$ or ${ }^{4} \mathrm{He}$ ) in coincidence with fission one has access to the fission probabilities for different $\mathrm{CN}$. For instance, with the same ${ }^{243}$ Am target, the CENBG-Argonne collaboration has determined the n-induced fission cross-sections on ${ }^{242} \mathrm{Cm},{ }^{243} \mathrm{Cm}$, ${ }^{244} \mathrm{Cm}$ et ${ }^{241} \mathrm{Am}$. This is illustrated in figure 2 . This also opens the possibility of a cross-check by means of different combinations of targets, beams and detected particles when they lead to the same $\mathrm{CN}$.

Such an inter-comparison is highly desirable. Indeed, beyond the usual mastering of the experimental challenges, the validity of the surrogate method relies on suppositions as well as on approximate theoretical foundation. These questions are analyzed for instance in refs [1-5]. They mostly concern whether, for the same excitation energy, the CNs formed by n-capture or by particle transfer are identical. Is their content not significantly affected by quantum numbers such as, for instance, angular momentum. This is all the most important because the surrogate method only yields de-excitation probabilities of the $\mathrm{CN}$ and must therefore rely on the theory of $\mathrm{CN}$ formation to generate cross-sections. Another assumption has to do with the de-excitation channels themselves. Generally, they are assumed to be correctly described by the WeisskopfEwing theory [6-8]. This theory is based on statistical considerations in which angular momentum does not play a role. Although experimental results (compare blue and red points in

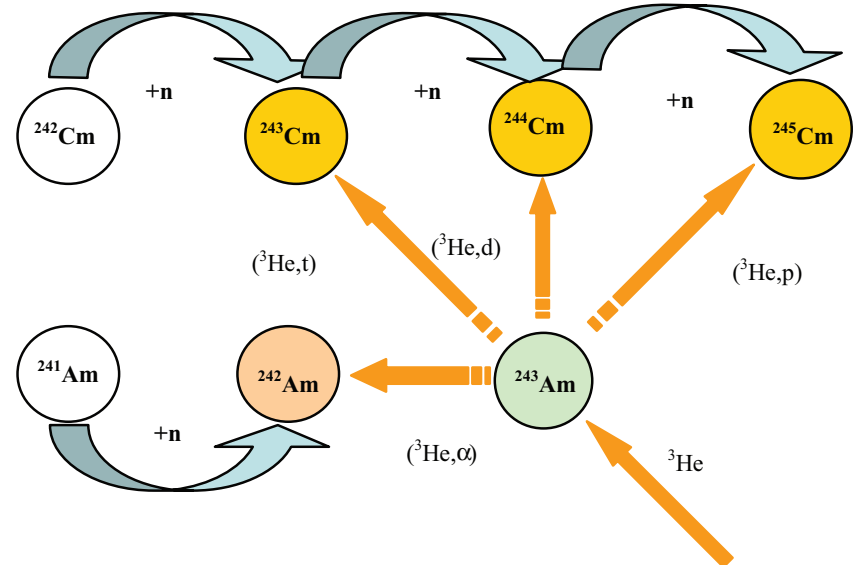

Fig. 2. Various experiments associated with a transfer reaction induced by the collision of a ${ }^{3} \mathrm{He}$ beam on a target of ${ }^{243} \mathrm{Am}$. Depending on the detected particle the $\mathrm{CN}$ systems are ${ }^{243} \mathrm{Cm}(\mathrm{t})$, ${ }^{244} \mathrm{Cm}(\mathrm{d}),{ }^{245} \mathrm{Cm}(\mathrm{p})$ et ${ }^{242} \mathrm{Am}(\alpha)$. Targets which would correspond for a direct $n$-capture experiment are ${ }^{242} \mathrm{Cm},{ }^{243} \mathrm{Cm},{ }^{244} \mathrm{Cm}$ et ${ }^{241} \mathrm{Am}$ respectively.

fig. 1) tend to yield credibility to the assumptions made in the surrogate method, the conditions of their validity must still be given careful attention.

Promises held by the surrogate method extend beyond the coherent and extensive study of n-capture cross sections in actinides whether it is for the less known thorium cycle or for the transmutation products of the U-Pu cycle. Intercomparison will provide tests of present theories of the $\mathrm{CN}$ formation. In all domains where the $\mathrm{CN}$ concept is valid, this method also holds a high physics potential. For instance, in the astrophysics of the s-process, the concerned reactions are difficult to assess by direct means. Recent advances in spectrometers open another opportunity by means of transfer reactions induced by heavy-ion beams. One may for example consider inverse kinematics transfer reactions induced by a ${ }^{238} \mathrm{U}$ nucleus on a light target such as ${ }^{12} \mathrm{C}$. Given the variety of final light systems (equivalents of p, d, t, $4 \mathrm{He}$ in the previous reaction), for the first time, one would be in position to determine fission fragments distributions as a function of excitation energy for $\mathrm{CN}$ such as ${ }^{239} \mathrm{~Np}^{*},{ }^{242} \mathrm{Am}^{*},{ }^{244} \mathrm{Cm}^{*}$. Moreover the simultaneous access to a much better known $\mathrm{CN}$ such as ${ }^{240} \mathrm{Pu}^{*}$ may provide the necessary cross-checks.

\section{Spallation}

As a theme in physics, spallation goes back to the first years following second world war (Serber, Fermi). However, the gradual split between the community interested in the physics of the nucleus and that working in particle physics led each to focus on experimental programmes which appeared the more promising to their specific goals. As a result, spallation remained sort of left in the middle. Thus, for almost twenty years the numerical simulation scheme devised and implemented by Bertini [22] staid a reference on the subject. A first revival of interest can be associated with the high energy heavy-ion collision programme at the BEVALAC in Berkeley 


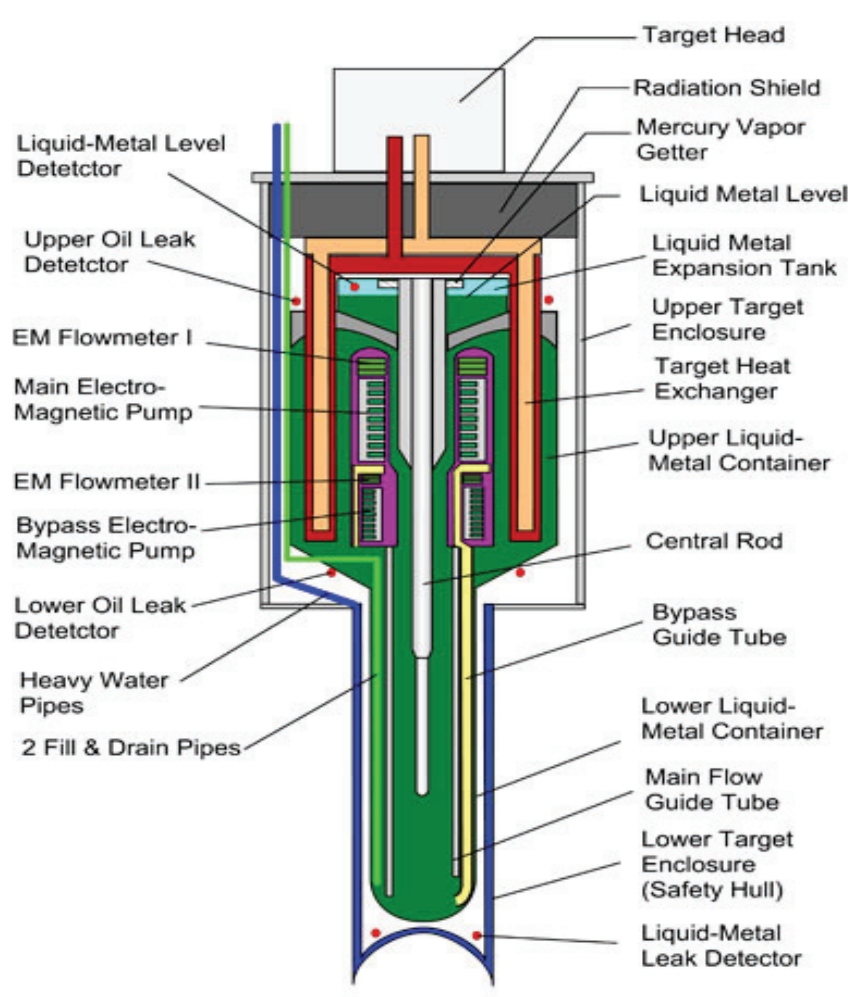

Fig. 3. Schematic view of the MEGAPIE target. The green area corresponds to the $\mathrm{Pb}$ - $\mathrm{Bi}$ eutectic fluid. The proton beam generated by the PSI cyclotron hits from bottom into the green finger which is the real target. The upper part hosts the pumps which circulate the fluid and ensure its cooling. On this drawing, the ratio of horizontal over vertical dimensions is enhanced.

at the turn of the 80's. At about the same time, spallation was selected as a promising way of generating neutrons for condensed matter science facilities. More recently, the proposal to use sub-critical accelerator driven reactors for the transmutation of minor actinides gave it another boost. The success of the MEGAPIE experiment at PSI [9] (fig. 3) has certainly shown that such systems are viable options when it comes to produce intense neutron fluxes. For what concerns us today, it also conveniently outlines two lines of investigation when spallation is considered as a process involving the collision of a proton and a single nucleus (fig. 4).

The first theme is the physics of neutron production by spallation in heavy nuclei such as $\mathrm{Bi}, \mathrm{Pb}$ or $\mathrm{W}$. The other theme concerns consequences which over time are considered to be potentially detrimental to the optimal operation of the target: the production of fragments of intermediate mass both in the eutectic and in the structure materials. An experimental programme started in the middle of the nineties using mostly the SATURNE facility and later the FRS spectrometer at GSI [11] has yielded a comprehensive coverage of both aspects of spallation [11-21].

It is in the first phase of the interaction of the proton with a nucleus that most of the energetic neutrons are produced. Based on works started in the eighties, new intra-nuclear cascade codes have been constructed which include in a more detailed manner effects associated with the Pauli principle, the production and the absorption of mesons and excited

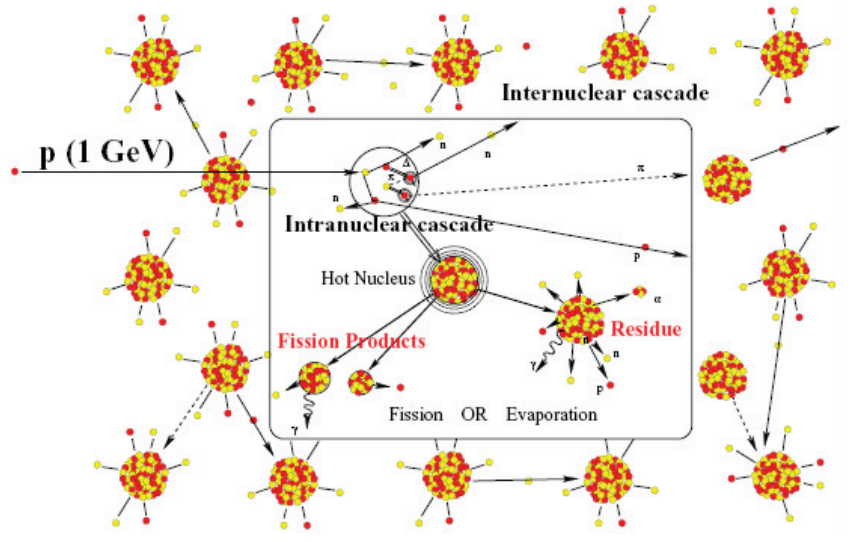

Fig. 4. Schematic picture of the spallation reaction. The zone outside the black rectangular frame corresponds to inter-nuclear phenomena in which residues (neutrons, fragments, gammas) from the spallation on one nucleus interact with another nucleus. Within the frame the figure illustrates the different phase of the spallation on a single nucleus. In present codes they are described sequentially, beginning with a cascade and ending with an evaporation phase.

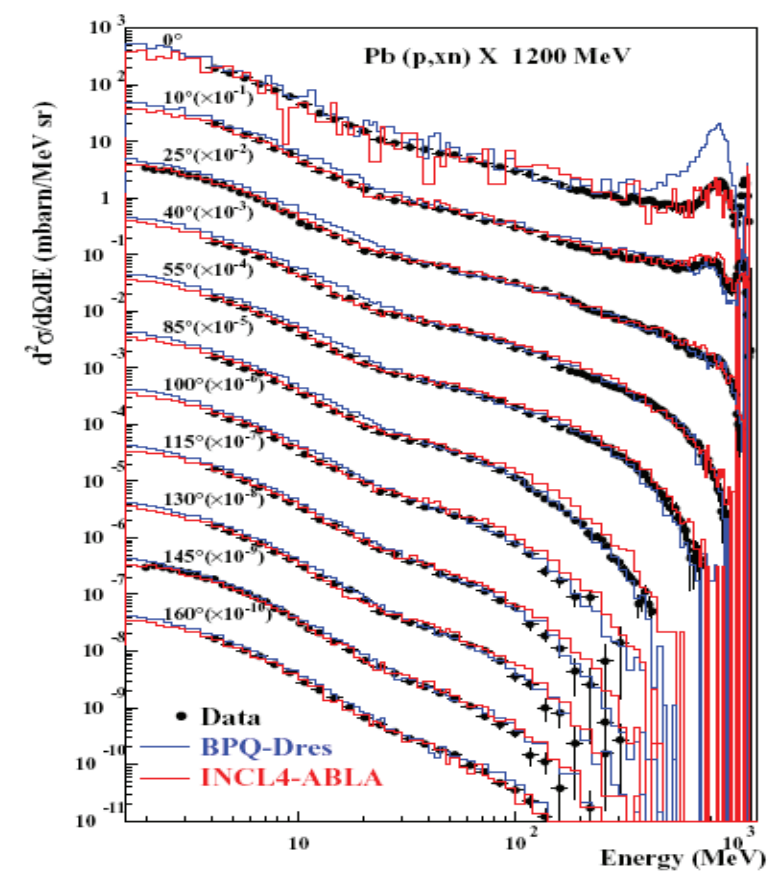

Fig. 5. Differential neutron production cross section as a function of the energy and the scattering angle of the emitted neutron for the reaction $\mathrm{p}+{ }^{208} \mathrm{~Pb}$ at $1 \mathrm{GeV}$.

baryons as well as the geometry of the heavy nucleus and its evolution as the collision develops in time. Such codes are very successful at describing the cascade phase [23,24]. Figure 5 illustrate the ability to explain the neutron production data for a complete set of nuclei of medium and heavy mass.

Once the cascade is completed, a significant fraction of the initial beam energy has been evacuated in the form of primary particle whose mass number rarely exceeds few units. Then the physical situation becomes more difficult to describe. It seems fair to say that this is still an open problem. While in the 
very last phase, evaporation models such as those considered in the previous section for the $\mathrm{CN}$ become valid [6], for the intermediate phase several models have been proposed. They could either be extensions of evaporation models based on improved nuclear level densities or on fragmentation models $[25,26]$. On the other hand, the GEMINI model $[27,28]$ which fairly well reproduces data in figure 6 defines fragment production as an extension of the fission process to excited light systems.
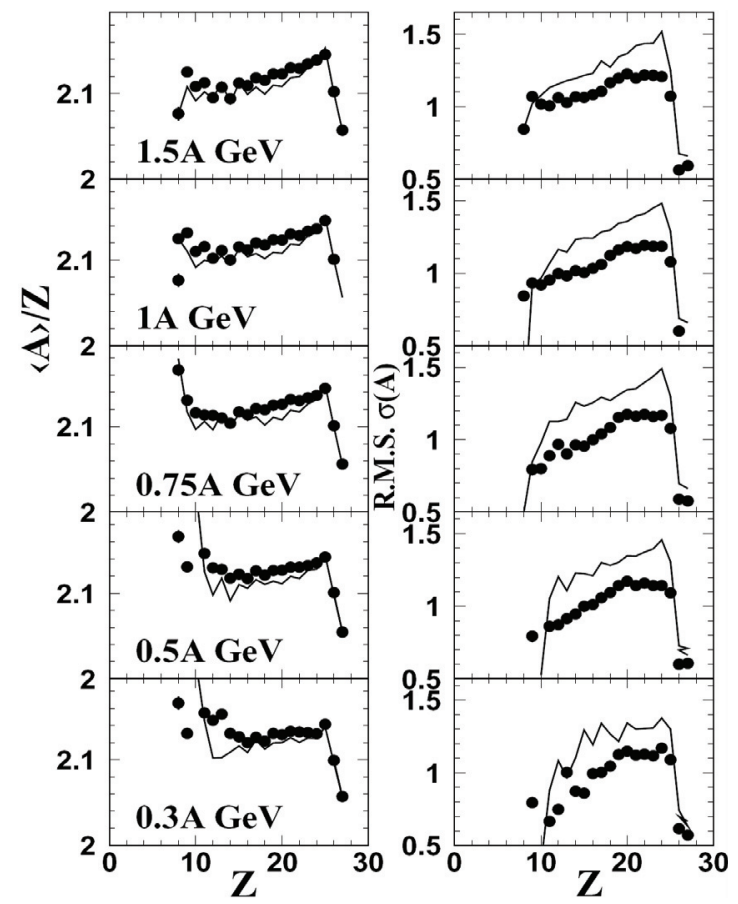

Fig. 6. First two moments of isotopic distributions of fragments following a spallation reaction on ${ }^{56} \mathrm{Fe}$ induced by protons of energy ranging from 0.3 to $1.5 \mathrm{GeV}$. The left part gives the average value of the mass and the left part its dispersion. Points correspond to experiment [29] while the solid lines are the predictions of a code which combines the INCL4 [24] cascade with the GEMINI [27,28] deexcitation model.

A full understanding of the mechanisms underpinning spallation would be extremely useful in spatial sciences and astrophysics. Indeed, it would allow accurate predictions of the effects of cosmic rays on materials in space whether of natural or artificial origin. Moreover, the de-excitation of nuclear systems at intermediate energy (second phase of spallation) is an important mechanism in the terminal phases of the r-process when elements ( $\mathrm{Pu}$ and beyond) are destroyed by intense fluxes of neutrinos, neutrons and gammas. The strong neutron excess in these heavy isotopes prevents such reactions to be experimentally studied in a foreseeable future.

\section{Advances in microscopic theory of fission}

For obvious reasons, fission has been and still remains a central problem for physicists involved in nuclear energy production. Still, despite the seniority of the enterprise, the

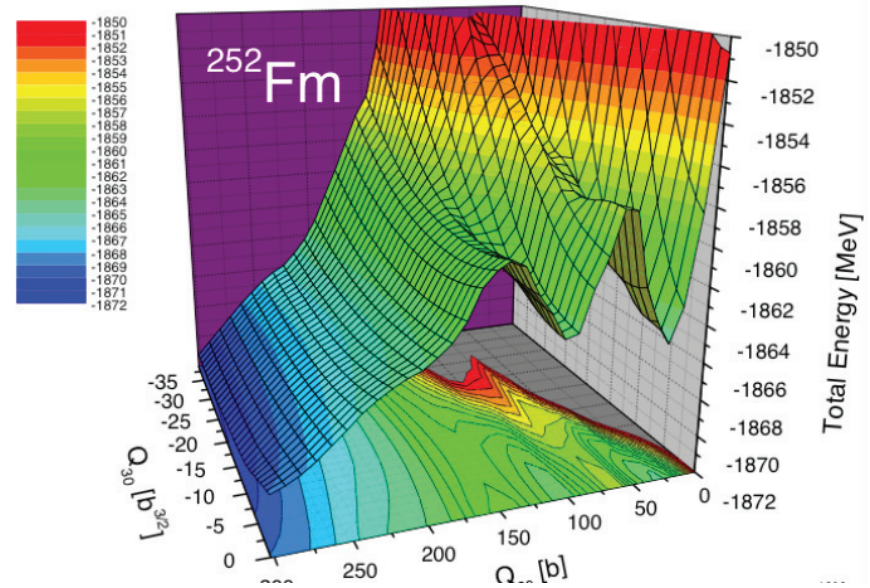

Fig. 7. Fission energy surface de fission of ${ }^{252} \mathrm{Fm}$ calculated with a Skyrme functional as a function of mass quadrupole and octupole moments $[34,35]$.

present description of the fission process remains largely dominated by phenomenology. In the last years of the sixties, the Nilsson-Strutinsky model provided an opening by allowing a semi-microscopic calculation of fission barriers. Few years later, advances in the definition of density functionals led to the first fully microscopic calculations $(\mathrm{HF}+\mathrm{BCS}$ and $\mathrm{HFB}$ methods) of the fission barrier of ${ }^{240} \mathrm{Pu}$ taking into account in a coherent manner, both the mean-field and the pairing component of the nucleon-nucleon effective interaction.

Until recently, further progress has been rather slow. In fact, what was needed was a density functional theory with a predictive capacity of a global nature (the full chart of isotopes, a large set of ground state and excited state properties). This long investigation if not concluded yet, seems at least to have reached a more satisfactory state. Microscopic theory is now in the position to predict masses and fission barriers with an accuracy as good as that of the most accurate phenomenologies [30-33].

In addition, the greatly enhanced computing power gives the possibility of fast and systematic calculations of fissionfusion energy surfaces as a function of multipole variables which take into account the complete set of shapes that a nucleus go through on its way to fission [34-37] (fig. 7).

As exciting such results may be, they still only correspond to half the solution. It is not enough to describe the static landscape over which fission takes place. One must also give a quantum description of the dynamics. It is only recently that encouraging results have been published $[36,37]$.

Figure 8 is one such example. It shows the distribution of fission products of ${ }^{238} \mathrm{U}$. Similar encouraging results have been obtained for fragment kinetic energies and half-lives. Thus, when experiment is out of reach, it seems now that exclusive reliance on phenomenology for prediction may not last.

However, there is still more work ahead. There are several approximations in the method of solution of dynamical equations. Success must be confirmed on a much more extended set of nuclei. Finally, there remains to build a full N-body theory of the fission process. Such a theory would avoid the recourse to collective variables. Indeed, however plausible their choice may be, they are still an ad-hoc physicist's input into the le microscopic description. Fission is the paradigm of 


\section{one-dimensional \\ dynamical \\ Wahl (experiment)}

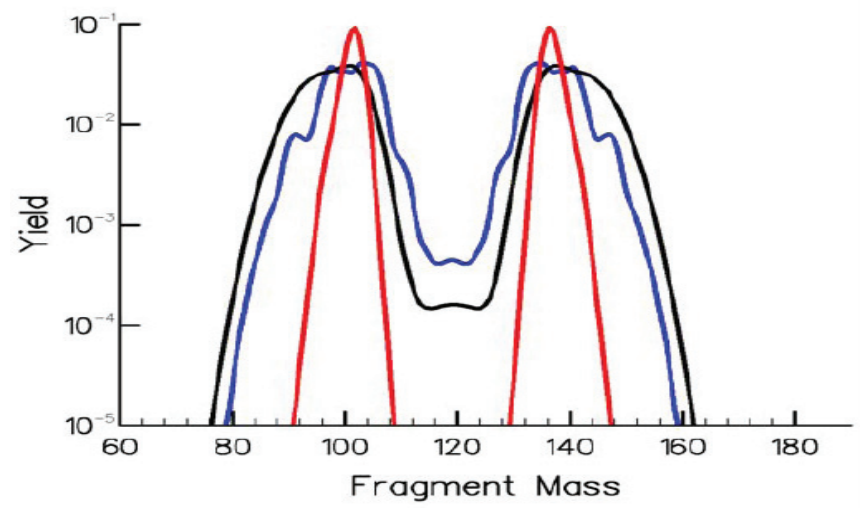

Fig. 8. Distribution of fission fragments of ${ }^{238} \mathrm{U}$. The red solid line corresponds to a one-dimensional dynamics along the fission valley bottom while the blue curve takes into account the full energy surface. The dynamics is computed with the Generator Coordinate Method [36].

the nuclear-large-amplitude-collective-motion. Any advance towards its solution will impact every aspect of that important subfield of nuclear structure for which collective motion is relevant.

\section{From actinides to superheavies}

Beyond the area of the chart of isotopes which is of direct interest to reactor physicists, Nuclear Theory has predicted the existence of a set of nuclei whose half lives rapidly decrease as the value of $Z$ grows. Over the years, some of these nuclei (up to $\mathrm{Z}=111, \mathrm{Rg}$ ) have been discovered in Berkeley, Dubna and Darmstadt [38-40]. There are many solid indications that the relative stability of these nuclei is caused by a strong prolate shell effect. Since the end of the sixties, an even more exciting prediction has been on the table: the existence of a stability island associated with new magic neutron and proton numbers beyond those of ${ }^{208} \mathrm{~Pb}$; the superheavy ( $\mathrm{SH}$ ) island.

The experimental search of $\mathrm{SH}$ nuclei has been a long and until recently unsuccessful story sometimes punctuated by premature announcements. Nevertheless, it seems that recent results obtained at Dubna now provide very strong evidences that SH nuclei have finally been observed. The signal still remains indirect. As for lighter elements, it relies on the repeated detection of chains of $\alpha$-decays with exceptionally large energies. Unfortunately, contrary to elements with $\mathrm{Z}$ between 104 and 111, these new chains are not connected to known data. This prevents an indisputable assignment of $\mathrm{N}$ and $\mathrm{Z}$ for the nucleus at the origin of the chain.

The same theories tested on the microscopic description of fission can be applied to the prediction of known $\alpha$-decay energies. The results are shown in the left-lower corner of figure 9. Such a global agreement certainly heartens one to make prediction as to what these energies would become

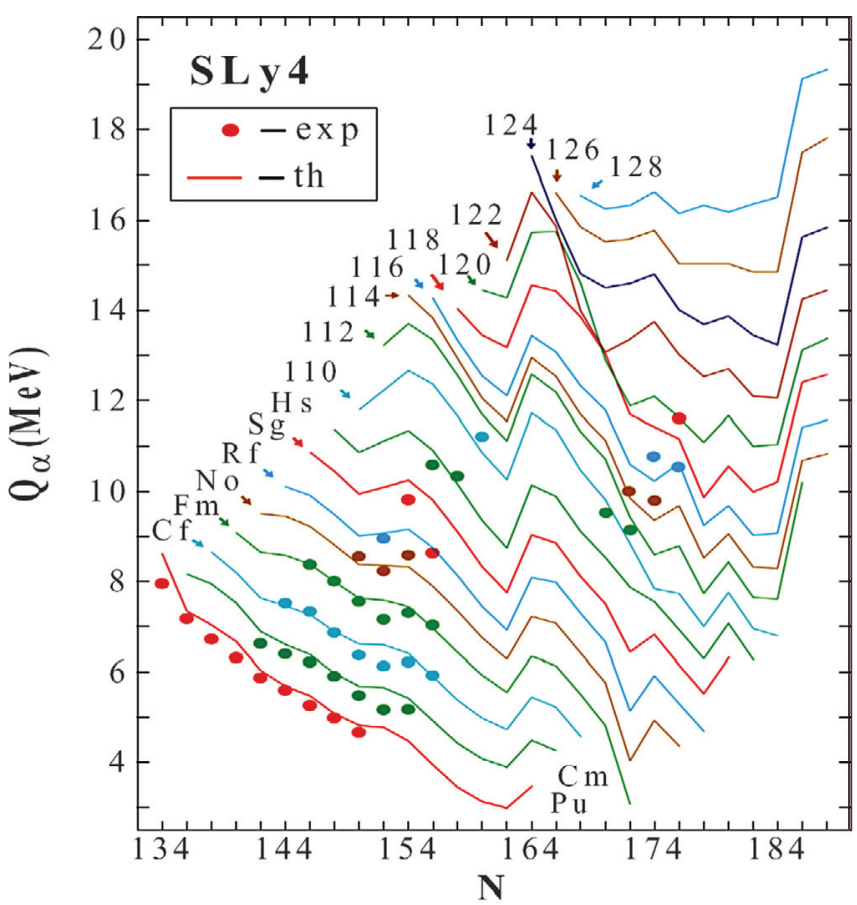

Fig. 9. Alpha decay energies for elements from actinides to superheavies. Solid lines correspond to the Skyrme density functional theory [41]. Points in the lower left corner are data for well identified nuclei. At the center of the figure, for values of $\mathrm{N}$ in the range [170178 ] points and $(\mathrm{N}, \mathrm{Z})$ assignments are those proposed by Dubna team on the basis of their data [40].

in the $\mathrm{SH}$ region corresponding to the Dubna results. The corresponding points on figure 9 can be said to provide another indirect supporting evidence.

On the other hand, the theoretical situation concerning magic numbers beyond $\mathrm{Z}=82$ and $\mathrm{N}=126$ is still unsettled. For instance, for the proton magic numbers three values of $\mathrm{Z}$ $(114,120,126)$ have been proposed [42-47]. This variability in prediction is especially interesting because it can be traced back to specific components of the density functional which are poorly constrained by data on that part of the chart which has been explored experimentally. These are the spin-orbit and the tensor parts. Just as n-rich exotic nuclei, superheavies are in position to provide decisive information on how to adjust these components of the functional.

As an illustration, the middle part of figure 10 shows the Nilsson spectrum of ${ }^{250} \mathrm{Fm}$ calculated with the Skyrme Sly4 force defined from neutron-matter calculations. It has no tensor term and the neutron and proton density dependence of its spin-orbit term is that of standard Skyrme forces. By contrast, the SkI4 force (lower part in figure 10) uses an isospin density dependence inspired by relativistic models. Finally the T44 force (upper part) is an attempt to introduce a tensor term into the Sly4 functional [42]. From top to bottom, the intensity of the $Z=114$ shell gap decreases while that of $\mathrm{Z}=120$ grows.

It turns out that such theoretical considerations on $\mathrm{SH}$ can be connected to data accessible in the region of heavy actinides. In a fermion system such as the nucleus, because of the Jahn-Teller effect, the most stable configurations are 


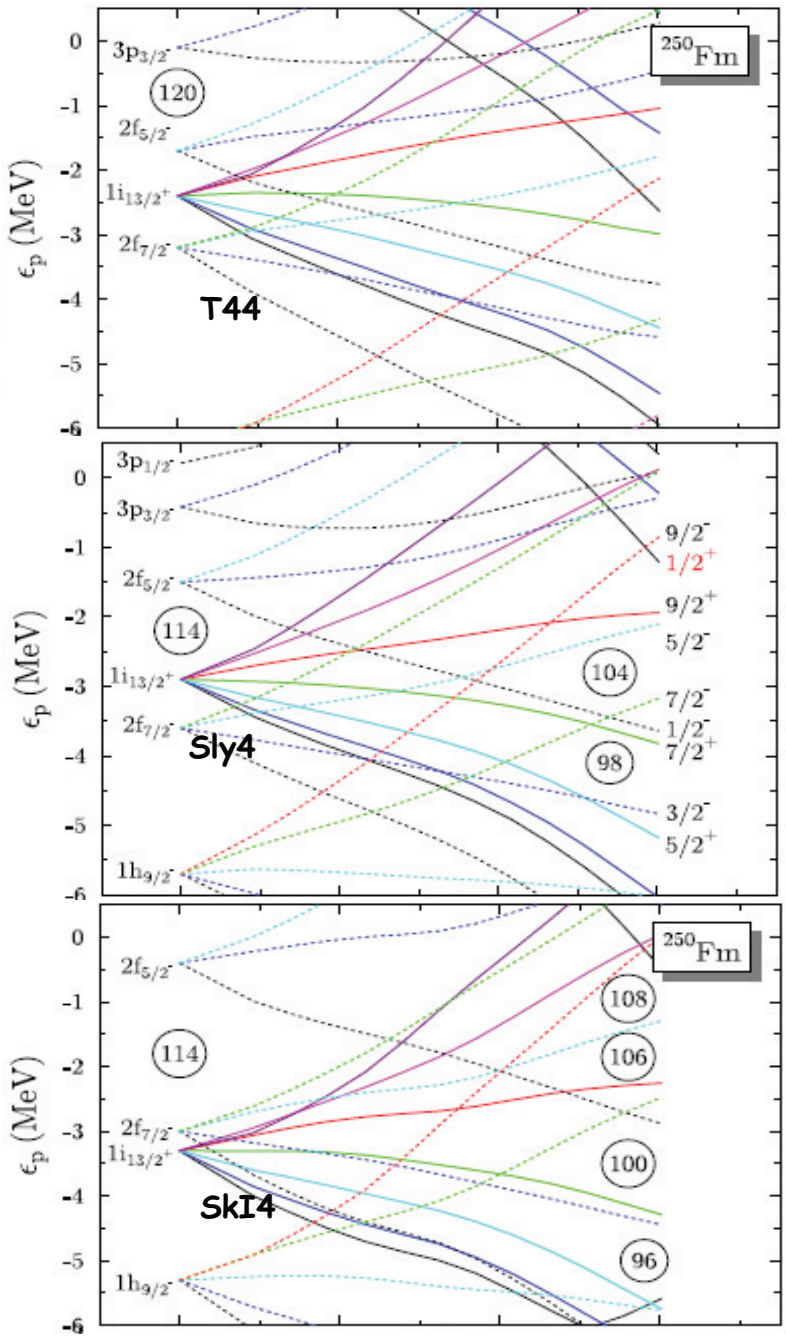

Fig. 10. Proton Nilsson single-particle spectrum as a function of quadrupole deformation calculated fot the nucleus ${ }^{250} \mathrm{Fm}$ with three density functionals SkI4, Sly4 and T44 (see text) [42].

those which minimize the single-particle level density at the Fermi surface. For that reason SH have a spherical density while heavy actinides are deformed. This is illustrated in figure 10. Because quadrupole deformation lifts the spherical single-particle degeneracy, some of the single-particle levels at the Fermi surface in a SH nucleus dive into the Fermi sea. They are thus present at the Fermi surface of actinides and therefore accessible to experimental investigation. The first promising results [48] have been obtained on isomeric states whose decay is inhibited because they are built on high angular momentum single-particle states. These states are precisely those mentioned above which are the most strongly affected by deformation and are found at the Fermi surface of both $\mathrm{SH}$ and Actinides. HFB calculations performed with the Sly4 and SkI4 forces [42] show that theoretical predictions of the excitation energy of isomeric states strongly depend on the structure and strength of the spin-orbit term. Based on such combined theory-experiment spectroscopic analyses on the actinides, one may thus envision to reach a physics beyond our present $(\mathrm{N}, \mathrm{Z})$ horizon and collect additional evidences on the structure of superheavy nuclei.

\section{Conclusion}

In a way, my selection of themes has only served to illustrate what is obvious to all of us: physics and experimental methods which underpin our understanding of the data required by Nuclear Energy is just a part of the corpus of basic nuclear science. The focus on the immediate needs of application and engineering, certainly gives a distinct character to this research. Nevertheless, it is never disconnected from the motivations of the most fundamental science which as much inspires it as it is inspired by it.

The author acknowledges a support from the inter-department programme of CNRS: PACEN (Programme pour l'Aval du Cycle et l'Énergie Nucléaire).

\section{References}

1. M. Petit et al., Nucl. Phys. A 735, 345 (2004).

2. S. Boyer et al., Nucl. Phys. A 775, 175 (2006).

3. W. Younnes et al., Phys. Rev. C 67, 024510 (2003).

4. W. Younnes et al., Phys. Rev. C 68, 034610 (2003).

5. J.E. Escher et al., Phys. Rev C 74, 054601 (2006).

6. V.F. Weisskopf et al., Phys. Rev. 57, 472 (1940).

7. F. Tovesson et al., Phys. Rev. Lett. 88, 062502 (2002).

8. F. Tovesson et al., Nucl. Phys. A 733, 3 (2004).

9. MEGAPIE collaboration, http://megapie.web.psi.ch/.

10. H. Geissel et al., Nucl. Instrum. Meth. B 70, 286 (1992).

11. S. Leray et al., Phys. Rev. C 65, 044621 (2002).

12. J.P. Meulders et al., HINDAS Final Report EU FIKW-CT-00031 (2005)

13. F. Rejmund et al., Nucl. Phys. A 683, 540 (2001).

14. J. Benlliure et al., Nucl. Phys. A 683, 513 (2001).

15. W. Wlazlo et al., Phys. Rev. Lett. 84, 5736 (2000).

16. T. Enqvist et al., Nucl. Phys. A 686, 481 (2001).

17. B. Fernandez-Dominguez et al., Nucl. Phys. A 747, 227 (2005).

18. L. Audoin et al., Nucl. Phys. A 768, 1 (2006).

19. J. Taïeb et al., Nucl. Phys. A 724, 413 (2003).

20. M. Bernas et al., Nucl. Phys. A 725, 213 (2003).

21. E. Casajeros et al., Phys. Rev. C 74, 044612 (2006).

22. H.W. Bertini, Phys. Rev. 131, 1801 (1963).

23. Y. Yariv et al., Phys. Rev. C 20, 2227 (1979).

24. A. Boudard et al., Phys. Rev. C 66, 044615 (2002).

25. L. Dresner, Oak Ridge Technical Report. ORNL-TM-196.

26. W. Hauser et al., Phys. Rev. 87, 366 (1952).

27. R.J. Charity et al., Nucl. Phys. A 483, 371 (1988)

28. L.G. Moretto, Nucl. Phys. A 247, 211 (1975).

29. S. Leray (private communication).

30. J.M. Pearson et al., Nucl. Phys. A 777, 623 (2006).

31. S. Goriely et al., At. Data Nucl. Data Tables 77, 311 (2001).

32. S. Goriely et al., Phys. Rev. C 66, 024326 (2002).

33. M. Samyn et al., Nucl. Phys. A 700, 142 (2002).

34. A. Staszczak et al., IJMP E 14, 306 (2005).

35. W. Nazarewicz (private communication).

36. H. Goutte et al., Nucl. Phys. A 734, 25 (2004).

37. H. Goutte et al., Phys. Rev. C 71, 024316 (2005).

38. P. Armbruster, Annu. Rev. Nucl. Part. Sci. 50, 411 (2000).

39. S. Hofmann, Prog. Part. Nucl. Phys. 46, 293 (2001).

40. Y. Oganessian et al., Phys. Rev. C 70, 064609 (2004).

41. S. Cwiok et al., Nature 433, 705 (2005).

42. M. Bender, P.H. Heenen (private communication).

43. T. Duguet et al., Nucl. Phys. A 679, 427 (2001).

44. M. Bender et al., Nucl. Phys. A 723, 354 (2003).

45. A. Afanasjev et al., Phys. Rev. C 67, 024309 (2003).

46. J.L. Egido et al., Phys. Rev. Lett. 85, 1198 (2000).

47. P. Reiter et al., Phys. Rev. Lett. 82, 509 (1999).

48. R.D. Herzberg et al., Nature 442, 896 (2006). 